\title{
Método pilates aplicado ao período gestacional: seus benefícios para o parto natural
}

\author{
Pilates method applied to the pregnancy period: its benefits for natural child bireth \\ Método pilates aplicado al período de embarazo: sus beneficios para el parto natural
}

Recebido: 29/10/2021 | Revisado: 04/11/2021 | Aceito: 09/11/2021 | Publicado: 14/11/2021

\author{
Cinara Araujo Felix \\ ORCID: https://orcid.org/0000-0002-5891-9509 \\ Centro Universitário da Amazônia, Brasil \\ E-mail: felixcinara@gmail.com \\ Nataly dos Santos Nascimento \\ ORCID: https://orcid.org/0000-0003-2865-9459 \\ Centro Universitário da Amazônia, Brasil \\ E-mail: natalynascimento468@gmail.com \\ Sheila Cristina Ribeiro de Almeida \\ ORCID: https://orcid.org/0000-0001-8163-5873 \\ Centro Universitário da Amazônia, Brasil \\ E-mail: sheila2016sophia@gmail.com \\ Naira Patrícia Castro de Oliveira \\ ORCID: https://orcid.org/0000-0003-1896-7693 \\ Universidade do Estado do Pará, Brasil \\ E-mail: nairacastro@outlook.com
}

\begin{abstract}
Resumo
Objetivo: Discorrer a respeito do método de pilates durante a gestação e os seus benefícios para um parto natural. Metodologia: Trata-se de uma revisão bibliográfico narrativa, realizada através de artigos com base científica de periódicos disponíveis nas bases de dados eletrônicas, como google academico, scielo, livros e revistas, pesquisas que abordam o método pilates no período gestacional que estejam nos anos de 2011 a 2021. Palavras chaves que foram utilizadas para a pesquisa: Método pilates, gestação, parto natural, método pilates, atividades físicas. Resultados: Os resultados que aqui se aplicam, sugerem que a prática de pilates, durante o período gestacional, levando em consideração as dimensões que este exercício atinge, são em suma, determinantes para o processo de parto, ao modo que, atende as necessidades de expansão do corpo, sugeridas ao organismo. Considerações Finais: O pilates, apresenta em seu quadro, uma complexa grade que atende as demandas do organismo de forma ampla, se estende a dores pontuais, e amplas.
\end{abstract}

Palavras-chave: Método pilates; Gestação; Parto natural; Atividades físicas.

\begin{abstract}
Objective: Discuss about the pilates method during pregnancy and its benefits for a natural birth. Methodology: This is a narrative bibliographic review, carried out through scientifically-based articles from journals available in electronic databases, such as google academico, scielo, books and magazines, researches that address the Pilates method in the gestational period that are in the years from 2011 to 2021. Key words that will be used for the research: pilates benefit, pregnancy, natural childbirth, pilates method. Results: The results that apply here, suggest that the practice of Pilates, during the gestational period, taking into account the dimensions that this exercise reaches, are, in short, determinants for the delivery process, as it meets the needs of expansion of the body, suggested to the organism. Final Considerations: Pilates presents in its frame a complex grid that meets the demands of the organism in a broad way, extending to punctual and ample pains.
\end{abstract}

Keywords: Pilates method; Gestation; Natural childbirth; Physical activities.

\section{Resumen}

Objetivo: Discutir sobre el método pilates durante el embarazo y sus beneficios para un parto natural. Metodología: Se trata de una revisión bibliográfica narrativa, realizada a través de artículos con base científica de revistas disponibles en bases de datos electrónicas, como google académico, scielo, libros y revistas, investigaciones que abordan el método Pilates en el período gestacional que se encuentran en los años de 2011 a 2021. Palabras clave que se utilizarán para la investigación: beneficio pilates, embarazo, parto natural, método pilates. Resultados: Los resultados que aquí se aplican, sugieren que la práctica de Pilates, durante el período gestacional, teniendo en cuenta las dimensiones que alcanza este ejercicio, son, en definitiva, determinantes para el proceso de entrega, ya que satisface las necesidades de expansión del cuerpo, sugerido al organismo. Consideraciones finales: Pilates presenta en su marco una retícula compleja que satisface las demandas del organismo de manera amplia, extendiéndose a dolores puntuales y amplios.

Palabras clave: Método pilates; Gestación; Parto natural; Actividades físicas. 


\section{Introdução}

No decorrer da gestação, a mulher acaba passando por grandes mudanças fisiológicas, anatômicas e bioquímicas em praticamente todos os sistemas. Isso começa nas primeiras semanas e prossegue até o término da gestação, sendo uma pequena parte destes, nos primeiros momentos do puerpério ou até a volta totalmente do organismo materno às circunstâncias prégravídicas (Burg, 2016).

De acordo com Nascimento e Mejia (2012), a gravidez tem relação com diversas mudanças musculoesquelética, quando as mulheres fazem o método de pilates como se fosse um exercício físico acaba promovendo vários benefícios para se ter um parto normal, tanto físico como emocional, pois, este método melhora a dor na área, fornece estabilidade ligamentar, articular e muscular, corrige a postura, fortalece e alonga totalmente os músculos, e relaxa bastante a gestante neste tempo.

No meio deste tempo de gestação, acontecem várias mudanças fisiológicas, a quantidade de hormônios se elevam essencialmente a progesterona e o estrogênio que são encarregados pela formação das situações perfeitas na gestão. Existe um crescimento no volume do sangue e nas mamas, onde o coração tem que bombear muito mais sangue para o corpo da gestante, na placenta e no corpo do bebe, isto é, os exercícios físicos são fundamentais para um aperfeiçoamento no condicionamento físico. O crescimento das mamas acaba ocorrendo uma sobre pressão bem maior no corpo, o que leva as gestantes a adotar uma postura de forma cifótica. A circulação pode atingir as válvulas que são as dirigentes para evitar o refluxo de sangue por conta do crescimento do sangue nas veias e nas artérias, deixando o sistema digestório mais devagar podendo ocorrer uma constipação e azia, além de sintomas mais comuns como os vômitos e enjoos (Burg, 2016).

O método de pilates é um planejamento do condicionamento físico e mental que utiliza uma técnica muito dinâmica para poder trabalhar com a força, flexibilidade, alongamento e equilíbrio, procurando permanecer as curvaturas fisiológicas do corpo, onde o abdome é o foco da força, no entanto, é trabalho todos o exercício desta técnica só que com repetições equilibradas (Martins, 2013).

Este método ajuda nas alterações que são provocadas pela gestação, além de trazer um bom conforto e benefícios para o parto natural, pois, estes exercícios além de gerar um fortalecimento, ajudam no alívio da dor na hora do parto e também destas mudanças que acontecem. Melhorando a circulação do corpo e principalmente do abdome que acaba sendo vantajoso para o bebê, ajudando para que a futura mãe tenha um pós-parto mais tranquilo (Martins, 2013).

Por conta destas diversas mudanças onde a maioria delas são hormonais, físicas e fisiologia que acontecem na gestação e as decorrentes apresentação e de dor e desconforto no parto natural, o presente projeto tem o objetivo em discorrer a respeito do método de pilates durante a gestação e os seus benefícios para um parto natural, por meio do levantamento bibliográfico e do estudo de caso feito com gestantes que fazem o pilates para ter um parto natural.

\section{Metodologia}

A presente pesquisa se destaca com o tipo Estudo Bibliográfico que compreende o levantamento de toda a bibliografia já publicada em forma de livros, periódicos (revistas), teses, anais de congressos, indexados em bases de dados em formato online. Sua finalidade é proporcionar ao aluno ou ao pesquisador o acesso à literatura produzida sobre determinado assunto, servindo de apoio para o desenvolvimento de trabalhos científicos e análise das pesquisas (Universidade Federal de Goias, 2008).

A pesquisa bibliográfica foi procedimento metodológico usado neste artigo. Conforme Gil (2002), a pesquisa bibliográfica tem que ser feita por meio de um material científico que já existe. Esta forma de pesquisa concede com que o investigador possui a cobertura de uma boa quantidade de elementos muito maior do que aquele que poderia ir à busca diretamente. Esta pesquisa bibliográfica permitiu o embasamento teórico relevante para o desenvolvimento dos objetivos de estudo. 
Para a produção deste artigo foram utilizadas bases de dados científicos como: Google acadêmico, na biblioteca eletrônica SCIELO, PubMed (Publicações Médicas) e BVS (Biblioteca Virtual em Saúde), no período de janeiro a agosto de 2020. Critério de inclusão: pesquisas que abordam o método pilates no período gestacional que estejam nos anos de 2011 a 2021, publicados em plataformas de dados científicos, na língua portuguesa e inglesa. Critérios de exclusão: pesquisas realizadas no ano anterior a 2011, monografias e artigos que não agregaram ao trabalhando em relação ao tema.

\section{Resultados e discussão}

Foram pesquisados 70 textos científicos, no qual foram excluidos 30 artigos que não se encaixavam com o tema Método pilates aplicado ao período gestacional: seus benefícios para o parto natural, e através da exclusão pela leitura foram excluidos 31 artigos. No total ficaram 9 artigos escritos na língua portuguesa e inglesa, a partir dos anos 2011 a 2021 , que se encaixam com o tema proposto.

Quadro 1 - Dados de autor (es), ano, objetivo (os), métodos/considerações.Santarém- Pa. 2021.

\begin{tabular}{|c|c|c|c|}
\hline Autores & Objetivo & Método & Resultados/Considerações \\
\hline $\begin{array}{c}\text { Freitas M.S } \\
2011\end{array}$ & $\begin{array}{l}\text { verificar a eficácia } \\
\text { do método Pilates } \\
\text { na diminuição da } \\
\text { dor lombar e na } \\
\text { melhora da postura } \\
\text { da gestante. }\end{array}$ & Relato de caso & $\begin{array}{l}\text { o método Pilates é eficaz na redução do quadro álgico lombar } \\
\text { apresentado durante a gestação; que houve o aumento gradativo das } \\
\text { curvaturas fisiológicas, inerentes à gestação; que não houve alteração } \\
\text { das curvaturas laterais da coluna vertebral, previamente observadas na } \\
\text { paciente; que houve uma melhora da consciência corporal e um } \\
\text { aumento da motivação em realizar atividades, relatadas pela paciente. }\end{array}$ \\
\hline $\begin{array}{c}\text { Quartarolo } \\
\text { Ingrid } \\
2017\end{array}$ & $\begin{array}{l}\text { Apresentar os } \\
\text { benefícios do } \\
\text { metodo pilates } \\
\text { praticado por } \\
\text { mulheres } \\
\text { gestantes. }\end{array}$ & Estudo descritivo & $\begin{array}{l}\text { O método pilates é diretamente ligado ao trabalho dos músculos } \\
\text { respiratórios sendo o mais importante o músculo diafragma que sofre } \\
\text { diretamente com o crescimento do feto e a diminuição da mobilidade } \\
\text { deste músculo. O trabalho respiratório ajudará a manter essa } \\
\text { mobilidade diafragmática que trará mais conforto principalmente no } \\
\text { final da gestação, além do controle das alterações emocionais e } \\
\text { ansiedade, comuns nessa fase }\end{array}$ \\
\hline $\begin{array}{c}\text { Nascimento D. } \\
2017\end{array}$ & $\begin{array}{l}\text { Descrever o } \\
\text { método pilates e } \\
\text { seus benefícios } \\
\text { para o parto } \\
\text { natural. }\end{array}$ & Estudo descritivo & $\begin{array}{l}\text { A prática de Pilates beneficia a futura mamãe, ajudando a manter uma } \\
\text { boa postura. E consequentemente fortalece o cinturão abdominal, } \\
\text { contribui e da força aos músculos mais profundos como o transverso e } \\
\text { o psoas. Uma inclinação pélvica excessiva pode gerar dores na zona } \\
\text { lombar, além de má postura. }\end{array}$ \\
\hline $\begin{array}{c}\text { Pinheiro M.S } \\
2018\end{array}$ & $\begin{array}{l}\text { identificar a } \\
\text { adesão de } \\
\text { gestantes } \\
\text { participantes de } \\
\text { um programa de } \\
\text { extensão a um } \\
\text { programa de } \\
\text { exercícios } \\
\text { domiciliares } \\
\text { baseados no } \\
\text { método Pilates. }\end{array}$ & $\begin{array}{c}\text { Pesquisa } \\
\text { exploratória } \\
\text { quanti- } \\
\text { qualitativo }\end{array}$ & $\begin{array}{l}\text { A amostra do presente estudo foi composta por } 4 \text { gestantes, primíparas, } \\
\text { com nível de escolaridade moderada e baixa renda familiar, que } \\
\text { aderiram às quatro semanas do programa de exercícios domiciliares } \\
\text { baseados no Método Pilates. No que refere ao período gestacional e os } \\
\text { sintomas osteomusculares, a intensidade de dor lombar se intensificou } \\
\text { no terceiro trimestre, sendo que as mesmas relataram esses } \\
\text { desconfortos com níveis moderado e de bastante desconforto. Através } \\
\text { desta pesquisa, foi possível verificar os fatores motivacionais e as } \\
\text { dificuldades encontradas para a realização dos exercícios domiciliares, } \\
\text { sendo que as questões de dificuldades mais citadas foram a falta de } \\
\text { tempo para realizar os exercícios mais de três vezes por semana, }\end{array}$ \\
\hline $\begin{array}{l}\text { Mota Amanda } \\
2018\end{array}$ & $\begin{array}{c}\text { Descrever os } \\
\text { beficios do pilates } \\
\text { na gestação }\end{array}$ & $\begin{array}{l}\text { Pesquisa } \\
\text { descritiva }\end{array}$ & $\begin{array}{l}\text { A prática do Pilates na gestação beneficia a futura mãe, ajudando a } \\
\text { manter a boa postura e o fortalecimento da região abdominal. A prática } \\
\text { também aliviará a dor nas costas trazendo mais energia para o dia a dia. } \\
\text { Além do fortalecimento do assoalho pélvico, é preciso trabalhar } \\
\text { também a sua mobilidade para que ele possa ter uma boa dilatação. } \\
\text { Esse tipo de exercício faz com que a gestante aprenda a controlar essa } \\
\text { região e trabalha a respiração, dois pontos muito importantes para o } \\
\text { trabalho de parto. }\end{array}$ \\
\hline $\begin{array}{c}\text { Pontes Catarina } \\
2019\end{array}$ & $\begin{array}{l}\text { Relatar o impacto } \\
\text { do pilates na } \\
\text { gestação e pós } \\
\text { parto }\end{array}$ & Estudo descritivo & $\begin{array}{l}\text { O aumento de peso e a mudança do centro de gravidade são as } \\
\text { alterações mais significativas e visíveis. O surgimento de uma lordose } \\
\text { progressiva, resultante destas modificações estruturais, pode } \\
\text { provocar dores lombares e pélvicas ao longo da gestação. A dor pode } \\
\text { piorar devido ao aumento da fraqueza muscular, à flexibilidade e } \\
\text { mobilidade articular deficientes, à má postura e às compensações que } \\
\text { as mulheres experimentam durante a gravidez. O fortalecimento dos } \\
\text { músculos abdominais e das costas pode minimizar esse risco. O pilates }\end{array}$ \\
\hline
\end{tabular}




\begin{tabular}{|c|c|c|c|}
\hline & & & $\begin{array}{l}\text { auxilia na Melhoria ou manutenção das capacidades físicas; auxílio } \\
\text { no controle do peso; redução do risco de diabetes gestacional em } \\
\text { mulheres obesas; melhorias ao nível do bem-estar psicológico. }\end{array}$ \\
\hline $\begin{array}{c}\text { Valeriano PA. } \\
2020\end{array}$ & $\begin{array}{l}\text { Apresentar como é } \\
\text { importante a } \\
\text { preparação do } \\
\text { parto com o } \\
\text { método pilates. }\end{array}$ & $\begin{array}{l}\text { Pesquisa } \\
\text { exploratória } \\
\text { qualitativo }\end{array}$ & $\begin{array}{l}\text { Não há um consenso sobre qual atividade física é a melhor durante o } \\
\text { período gestacional No entanto, o American College of Obstetricians } \\
\text { and Gynecologists recomenda que devem ser exercícios de intensidade } \\
\text { regular e moderada, com as atividades centradas nas condições de } \\
\text { saúde da gestante, na experiência em praticar exercícios físicos e na } \\
\text { demonstração de interesse e necessidade da mesm Sendo assim, o } \\
\text { método Pilates é uma excelente escolha de atividade física durante o } \\
\text { período gestacional }\end{array}$ \\
\hline $\begin{array}{c}\text { Jeanne Carvalho } \\
2021\end{array}$ & $\begin{array}{l}\text { Descrever os } \\
\text { benefícios do } \\
\text { método Pilates } \\
\text { durante o período } \\
\text { gestacional, diante } \\
\text { das modificações } \\
\text { físicas. } \\
\end{array}$ & Relato & $\begin{array}{l}\text { O Pilates para gestantes vai colaborar muito para manter um corpo } \\
\text { forte e saudável para passar por todas as alterações que ocorrem nessa } \\
\text { fase tão única e ademais vai trabalhar a resistência muscular, que é } \\
\text { necessária para o trabalho de parto, principalmente os mais longos, e } \\
\text { também será de extrema importância para diminuir a ansiedade que as } \\
\text { acompanham desde o início da gestação e tendem a aumentar quando o } \\
\text { parto está chegando. }\end{array}$ \\
\hline $\begin{array}{c}\text { Andrade DC, } \\
2021\end{array}$ & $\begin{array}{l}\text { Apresentar a } \\
\text { importância do } \\
\text { fortalecimento do } \\
\text { assoalho pélvico } \\
\text { em gestantes. }\end{array}$ & $\begin{array}{c}\text { Estudo } \\
\text { Qualitativo }\end{array}$ & $\begin{array}{l}\text { Um fator predominante nos estudos foi que força do assoalho pélvico é } \\
\text { crucial para qualidade do parto. Além disso, foi percebido um } \\
\text { desconhecimento da atuação da fisioterapia em obstetrícia. O uso de } \\
\text { exercícios perineais na gestação trouxe maior força muscular e } \\
\text { colaborou para o trabalho de parto em primíparas no pós-parto normal. }\end{array}$ \\
\hline
\end{tabular}

Fonte: Autores (2021).

De acordo com os resultados do presente estudo, sugere-se que o método Pilates auxilia na redução da dor causada pelas alterações fisiológicas gestacionais, e que a oscilação do centro de massa de mulheres gestantes não é influenciada pelos exercícios do método.

Para Carvalho (2021), durante a gravidez, o corpo da mulher sofre diversas modificações e na maioria das vezes, é difícil o corpo estar preparado para suportar essas mudanças que irão acontecer em um curto espaço de tempo, em média 42 semanas. Por esse motivo, é necessário que se prepare o corpo da melhor forma possível. E o método Pilates vem com uma ótima opção para ajudar a gestante nessa preparação. O Pilates fortalece a musculatura, melhora a consciência corporal e ajuda no controle emocional. O que implica em amenizar os efeitos negativos durante a gestação.

A gestante que pratica atividades físicas estará menos propensa a ter dificuldades na hora do trabalho de parto e pósparto, visto que os exercícios elaborados para a mesma, trabalham músculos que serão recrutados na hora do parto como o fortalecimento da musculatura do assoalho pélvico que durante a gestação afetam a função visceral, intestinal e sexual, desempenhando também um importante papel na sustentação dos órgãos internos (Pinheiro, 2018).

Em consonância Mota (2018), discorre que cada trimestre da gestação pode ser trabalhado com foco diferenciado, pensando em suprir as necessidades de cada período. No primeiro trimestre ocorrem os constantes enjoos, assim como o aumento do peso e das mamas. Já no segundo trimestre, com o crescimento da barriga, o centro de gravidade começa a se deslocar pra frente aumentando a lordose e consequentemente a dor nas costas. O último trimestre traz com ele o cansaço e a distensão dos músculos da pelve.

A gestante que pratica Pilates melhora a qualidade do sono, o que consequentemente aumenta a energia diária, ao fortalecer os músculos pélvicos, o parto, em si, é facilitado, pois a futura mamãe aprende a controlar a zona pélvica. Certamente há um aumento na resistência física, alcançando maior agilidade e controle do peso, que se ganha devido à situação. Aprende-se a respirar melhor, de maneira profunda e controlada. Este tipo de respiração é o indicado para a hora das contrações e do próprio parto (Nascimento, 2017).

Segundo Quartorolo (2017), concentrar-se no treino dos músculos essenciais para a postura proporcionará a estabilidade postural e resistência dos músculos do assoalho pélvico, que ajudarão a diminuir as alterações posturais que 
podem causar dor lombar e disfunções do assoalho pélvico, como a incontinência urinária. Além disso, auxilia no desenvolvimento da resistência nos músculos estabilizadores da coluna, o que contribui para diminuição da dor lombar.

Valeriano (2020), indica que sempre devemos dar início pela ativação de assoalho pélvico, em seguida, transverso do abdômen, este último será de extrema importância na fase de expulsão do parto e também na rápida recuperação da musculatura abdominal. Os retos abdominais serão acionados enquanto puderem, e os oblíquos também, porém a nossa ênfase maior deve ser nos dois citados primeiro. Que serão ativados até o último trimestre. Nas últimas semanas, a partir de 34 semanas, podemos solicitar para que elas esqueçam aquela contração de assoalho pélvico e foquem no relaxamento.

Andrade (2021), descreve as técnicas que são mais utilizadas em gestantes no método pilate: Mobilidade de Coluna com Flexão e Rotação, The cat, Liberação e mobilização pélvica, Fortalecimento de membros superiores, Rosca bícepes com faixa elástica, Bridge com fitball e Agachamento unipodall. O pilates, apresenta em seu quadro, uma complexa grade que atende as demandas do organismo de forma ampla, se estende a dores pontuais, e amplas, sendo um dos instrumentos mais procurados pelas gestantes, por apresentar um esforço fisico menor, porém um condicionamento maior.

Ao modo que, o pilates apresente uma dimensão que atua diretamente ao físico do indivíduo, trabalhando aspectos de flexibilidade, alongamento, respiração, todas as dimensões necessarias que adentram o parto natural.

No relato de caso de Freitas (2011), foi possivél analisar a redução do quadro álgico lombar apresentado durante a gestação e que houve o aumento gradativo das curvaturas fisiológicas, inerentes à gestação. Que não houve alteração das curvaturas laterais da coluna vertebral, previamente observadas na paciente. Que houve uma melhora da consciência corporal e um aumento da motivação em realizar atividades, relatadas pela paciente.

Pontes (2019), trás em seu estudo a relação do pilates durante a gestação e seus inumeros benefícios pós parto dispõe que nessa fase, é importante voltar a equilibrar o corpo depois de todas as alterações sofridas na gravidez. Deve ser feita a avaliação da diástase abdominal (separação dos retos abdominais) e pode-se começar a pensar no trabalho a realizar. E o Pilates vai proporcionar uma atividade tranquila, consciente e gradual na recuperação do corpo após o parto, sendo realizado através de exercícios específicos para o fortalecimento muscular de todo o corpo, para além de exercícios de mobilidade.

Desta forma, os resultados que aqui se aplicam, sugerem que a prática de pilates, durante o periodo gestacional, levando em consideração as dimensões que este exercício atinge, são em suma, determinates para o processo de parto, ao modo que, atende as necessidades de expansão do corpo, sugeridos ao organismo.

\section{Considerações Finais}

O objetivo proposto nesse estudo, foi revisar na literatura, benefícios do método Pilates durante o período gestacional e como auxilia no processo de aderência ao parto natural os resultados mostraram que foi possível verificar que por meio da prática do método Pilates como contração do períneo, elevação de perna, ponte, gato arrepiado, saldação ao sol e alongamento de pernas. Voltado para a gravidez os movimentos precisam ser mais lentos, exige bastante concetração e precisão de movimento, além disso é muito importante a contração dos músculos do pavimento pélvico, pois melhoram o tônus a irrigação sanguinea e consequentemente a perda de urina.O estudo também evidência que o método pilates oferece benefícos na preparação do corpo dessa gestante, auxilia no processo de diminuição de dor lombrar, dor nas costas, fortalece o assoalho pélvico, ajuda no equilíbrio hormonal, além de proporcionar um pós parto muito mais prático.

È importante salientar, que apesar de ter alguns estudos sobre o tema, podemos observar que o método é pouco explorado, e que é necessário mais profissionais aplicando na prática o método pilates, e evidenciando seus beneficios para o parto e pós parto, quanto mais profissionais se empenharem em publicar estudos mais mulheres seram beneficiadas. 


\section{Referências}

Amorim, T. (2021) A importância do controle respiratório no pilates. Blog Pilates. https://blogpilates.com.br/controle-respiratorio-no-pilates/.

Andrade D. C. (2021). Importância do fortalecimento do assoalho pélvico em gestantes.

Burg, J. L. (2016). Os benefícios do pilates na gestação. FAEMA. http://repositorio.faema.edu.br

Chistófalo, C. M., Ariane, J., \& Tumerelo, A. S. (2003) A prática de exercício físico durante o período de gestação. E.F Deportes. com, 9 , 59.

Daminni, M. C. V. et., al (2019). mudanças na vida e no corpo da mulher durante a gravidez. Brazilian Journal of Surgery and Clinical Research - BJSCR. 27(1), 126-131.

Carvalho, J. (2021). Pilates na gravídez: Como a técnica ajuda na preparação do corpo para o parto.

Freitas, M. S., et al (2011). O método pilates na diminuição da dor lombar em gestantes. Goias.

Gil, A. C (2002). Como elaborar projetos de pesquisa

Kitechen, S., \& Bazin, S. Eletroterapia de Clayton. 10. ed. São Paulo: Manole. 1998. 350p. Marinho, A. C. N. Atuação fisioterapêutica no aleitamento materno e na intercorrência mamária. Fisioterapia Brasil. São Carlos, (4), 317-320.

Kroetz, D. C. (2015). Benefícios do método pilates nas alterações musculoesqueléticas decorrentes do período gestacional. Revista Visão Universitária

Marconi, M. de A., \& Lakatos, E. M. (2018). Técnicas de pesquisa: planejamento e execução de pesquisa, amostragens e técnicas de pesquisa, elaboração, análise e interpretação de dados.

Martins, R. A. S (2013). Método pilates e seus benefícios nas alterações osteomusculares do período gestacional. Monografia apresentada em Pós-graduação em Ortopedia e Traumatologia com Ênfase em Terapia Manual - Faculdade Ávila

Mota, A. (2018). Pilates na gestação. https://www.dafnemacruz.com.br/posts/m.php?l=pilates-na-gestacao.

Nascimento, T. G.M., \& Dayana, P. M (2021). O método pilates aplicado à ginecologia obstetrícia: benefícios durante o período gestacional e para o parto natural.

Neto, A. A. L (2018). O desenvolvimento fetal influenciado pela prática de exercícios físicos por gestantes.

Oliveira, A. C. P. M., Sene, L. B., \& Watanabe L. A. R. (2018). Percepção de dor no parto normal em gestantes.

Nascimento, D. (2017). Período gestacional e a importância do método pilates.

Valeriano, P. (2021). Preparação do parto com o método pilates para gestantes. 2021. http: pilates-na-gravidez-como-a-tecnica-ajuda-na-preparacao-do-corpopara-o-parto.html

Pereira, N. S., et al (2020). Os beneficios do método pilates diante das alterações no período gestacional. 2(4). Revista Cathedral

Pontes, C. (2019). Pilates na gravidez e no pós parto. http/blogs/oito-um/pilates-na-gravidez-pos-parto.

Pinheiro, M. S (2018). Adesão de gestantes participantes de um projeto de extensão a um programa de exercícios domiciliares baseado no método pilates. Biblioteca da universidade Feevale.

Quatarolo, I. (2017). Pilates conteporâneo para gestantes. https://www.pilatescontemporaneo.com/ebooks/Ebook-PilatesGestantes.pdf

Sedicias, S. É normal sentir falta de ar na gravidez? Tua Saúde. Online. https://www.tuasaude.com/falta-de-ar-na-gravidez/. A

Silva, R. (2018). Atuação do fisioterapeuta no período gestacional: uma revisão integrativa de literatura.

Souza T. k. R, Vieira, R. P, \& Neto A. A. L. (2018). Anais da III Jornada de Educação Física do Estado de Goiás: Corpo, ciência e mercado: os desafios para a Educação Física. https://www.anais.ueg.br/index.php/jefco/index.

Souza P. C. et al. (2019) Percepções sobre o pilates: do pré-natal ao pós parto. Saúde \& Transformação Social/Health \& Social ChangeSanta Catarina, Brasil. 